Please share your stories about how Open Access to this article benefits you.

\title{
Making It Easier To Be Green: A Single Case Demonstration of the Effects of Computer Defaults To Conserve Energy in a University Computer Lab
}

\author{
by Jason M. Hirst et al.
}

2013

This is the published version of the article, made available with the permission of the publisher. The original published version can be found at the link below.

Jason M. Hirst, Derek D. Reed, Brent A. Kaplan, and Jonathan R. Miller. (2013). Making It Easier To Be Green: A Single Case Demonstration of the Effects of Computer Defaults To Conserve Energy in a University Computer Lab. Sustainability: The Journal of Record 6(6): 340-344.

Published version: http://www.dx.doi.org/10.1089/SUS.2013.9827 


\title{
Making It Easier To Be Green: A Single Case Demonstration of the Effects of Computer Defaults To Conserve Energy in a University Computer Lab
}

\author{
Jason M. Hirst, MA, Derek D. Reed, PhD, BCBA-D, Brent A. Kaplan, BGS,
}

and Jonathan R. Miller, MA, BCBA

\begin{abstract}
Educational buildings and university campuses represent some of the most computer-dense settings in the United States. Unfortunately, the administrators and users in these settings often lack proper energy savings strategies, resulting in excessive energy waste. Research in behavioral economics has reliably shown that effort is an inhibitory factor in changing a variety of behaviors. That is, humans have a tendency to choose the option that requires the least amount of effort, regardless of whether that option is the best one. Thus, it might be inferred that interventions requiring greater effort for computer users to conserve energy are unlikely to be effective.
\end{abstract}

This study highlights a successful cost-cutting application of default energy savings settings in a campus computertesting laboratory. Default settings applied by the research team did not require effort on the part of users and resulted in computers powering-down after a relatively short period of inactivity. A cost analysis revealed modest fiscal and electricity savings among the small number of computers included in the study. However, extrapolating these modest savings across the many hundreds of workstations typically found on university campuses suggests a substantial savings would result from the adoption of the intervention described herein. Implications for practice and future research are discussed.

\section{Introduction}

Energy consumption has been a target of behavioral approaches to sustainability (e.g., behavior analytic, behavioral economics, behavioral psychology) for decades. ${ }^{1,2}$ Despite the increasing proliferation of personal computers (PCs) in commercial settings, relatively little behavioral research has been conducted on these devices. This paucity of research is troubling given the immense numbers of PCs present in commercial and public settings, such as university campuses or office buildings. The 2002 U.S. Department of Energy's Commer- cial Buildings Energy Consumption Survey $^{3}$ found that education, office, and health care buildings feature the highest density of PCs per square foot. Unfortunately, analyses conducted by Webber and colleagues ${ }^{4}$ found that walk-throughs of 12 office buildings (four of which were educational settings) indicated 60 percent of PCs were left on during overnight hours. Of the remaining 40 percent of PCs, 36 percent were turned off and only 4 percent were set to low power settings. These data complement a review of surveys ${ }^{5}$ of both commercial and residential PC users that found 90 percent of PCs are turned on for more than 40 hours per week (these computers are termed continuous-use machines). The survey data also indicated that continuoususe machines are powered on for an average of 152 hours per week, despite usage averaging only 40 hours per week, suggesting an average of 112 hours of energy wasted per week. These data collectively indicate a well-suited area for behavioral research on energy conservation in PC-dense settings.

A review of the literature suggested several factors that influence the effectiveness and success of energy conser-

Department of Applied Behavioral Science, University of Kansas, Lawrence, Kansas. 
vation initiatives. First, conservation efforts should avoid limiting choice or freedom (i.e., voluntary curtailment of energy use). Additionally, strategies are more likely to be effective when they limit the costs to consumers in terms of money, time, or physical or mental effort. ${ }^{6}$ A paper by Gifford ${ }^{7}$ referred to this phenomenon as tokenism. People tend to choose the easiest actions to adopt rather than the action with the largest impact on the environment.

Relevant research in behavioral economics has shown that regardless of which is the best option, humans have a tendency to choose whichever option requires the least or no effort (i.e., the default option). ${ }^{8,9}$ In sum, research has shown that effort is an important variable in human conservation behavior.

Applying what behavioral psychology knows about effort to the problem of reducing PC energy consumption, we can hypothesize that people fail to turn off their computers due to an increased effort in closing applications, saving documents, and shutting down the machine. PCs commonly have built-in functions to automatically power down after a period of inactivity, which would prevent continuous use, but that, too, requires time and effort as well as knowledge of how to manipulate these settings. Additionally, computers owned by institutions and organizations often have centrally managed system settings and deny individual users the administrative rights to make changes to computer settings or even shut down the computer, which may prevent the utilization of energy-saving mechanisms. It is likely the case that failures to reduce energy consumed by PCs is related to a lack of prompts in the environment to do so, a lack of motivation, and an avoidance of effort.

Using Van Raaij and Verhallen's behavioral interpretation of energy use from an economic perspective, ${ }^{10}$ the behaviors of concern in the continuous powering of PCs (i.e., energy-inefficient use) may be considered both (a.) PC operation and (b.) manipulation of PC power settings. As described, effort may be a prime variable in energy-inefficient PC use. Behavior analysts have long understood that when the consequences for a certain behavior are difficult to control, reducing the effort required for the desirable option can be a viable treatment option. ${ }^{8}$

More specifically, if an active behavior is difficult to promote or train through other means, capitalizing on inaction through the use of default options is a way to structure situations in which sustainable choices require less effort than unsustainable ones. Such an approach may yield a resource-efficient means of changing conservation outcomes. This study was undertaken to evaluate the effects of default energy conservation settings on PCs in a computer-testing lab on a university campus in an attempt to decrease energy consumption by minimizing the mental and physical effort required from users.

\section{Methods}

\section{Participants and Setting}

The participants in the study were 175 undergraduate students enrolled in an introductory-level course in behavioral science and seven undergraduate teaching assistants. Students in the course were required to use a computer-testing lab on campus outside of regular course time to take computerized quizzes. Course requirements consisted of two quizzes per week; each student was allotted two hours per week in the testing lab to complete the quizzes. One undergraduate teaching assistant was present to supervise the lab each hour, with up to 12 students allowed to take quizzes per hour. All participants were blind to the study during the entire semester.

The testing lab was a large room measuring nine meters by six meters containing 18 Dell $^{\circ}$ OptiPlex computers equipped with 17 -inch flat-panel monitors, and standard keyboards and computer mice. The testing lab was open Monday through Thursday and hours varied by day. The lab was open 12 hours per day on Mondays and Wednesdays, and nine hours per day on Tuesdays and Thursdays. Students were restricted to taking quizzes on one of 12 computers. Computer stations were divided into dyads where dyads were arranged by shared tables, such that two computers on the same table comprised one dyad. For this study, the energy consumption of four dyads was measured. The dyads were further divided into two groups (Groups 1 and 2; each group consisted of two dyads for a total of four computers) to permit comparisons between energy use during the four conditions described. (See Experimental Design and Procedure.)

\section{Apparatus and Dependent Measures}

The primary dependent measure collected to measure energy consumption was kilowatt hours (kWh) used per week. Data were collected using Watts Up? $\mathrm{PRO}^{\mathrm{Tw}}$ AC power meters (model 99333) available commercially from Electronic Educational Devices, Inc (Denver, CO). The utility and reliability of these meters was confirmed in a previous study. ${ }^{11}$ The meters were capable of recording data at preset intervals and storing the data using internal memory. The data were retrievable using a cable and program supplied by the manufacturer. Four meters measured energy consumption throughout the room. Each meter measured the energy consumption of one dyad of desktop computers (both computer processing units and monitors) by plugging the power cables of the computer devices into a power strip first, then plugging the power strip into the meter located under the desk and out of students' sight. The energy consumption of computers in the testing lab was extrapolated to calculate the cost to the institution of operating a 20-computer lab (the average size of labs at the present institution) per semester using the rate of $\$ 0.065$ per $\mathrm{kWh}$ (according to university records).

\section{Experimental Design and Procedure}

Given our interest in evaluating the effects of a specific intervention over time, we employed an experimental time-series analysis termed the multiple-baseline design. ${ }^{12}$ In the multiplebaseline design, treatment conditions are withheld across target participants/ settings for a baseline period. Upon observation of a stable dependent variable, the independent variable (IV) (e.g., treatment) is systematically introduced in a staggered fashion across the target participants/settings. 
Experimental control is established by demonstrating that the introduction of the independent variable only modifies the dependent variable for the participant/setting in which the treatment is in place, that is, the introduction of the IV for one participant/setting while the other(s) remain in baseline. Upon demonstration of a stable dependent variable for the first participant/setting with the treatment in place, the next participant/setting moves to treatment, and so on. For additional demonstrations of experimental control, the multiplebaseline design can be supplemented with reversals-the systematic removal and reintroduction of treatment conditions to effectively turn on and off the treatment effect. Note that this experimental design has been established as a viable research approach in sustainability studies. ${ }^{13,14}$

A multiple-baseline design across groups of dyads with reversals between conditions was used to compare energy consumption in the testing lab under four conditions. The researchers programmed the meters to record data at four-hour intervals. Each week, researchers retrieved data from the devices using manufacturer-supplied software. Although meters collected data each day of the week, data analysis was restricted to 8:00 a.m. Monday to 8:00 a.m. Friday to reflect periods designated for computer lab use. The lab's usage varied on Fridays and weekends, so to remove this potential extraneous variable, data were restricted to times during which a consistent level of use was maintained across weeks.

Baseline. The baseline condition evaluated the level of energy consumed by computers in the testing lab under original power management settings as programmed by university IT staff. In this condition, neither the computers nor monitors were set to enter standby mode (low-power state) automatically. However, users could manually set computers to standby mode during this and all subsequent conditions.

Default Condition. The purpose of this condition was to measure the amount of energy consumed by computers in

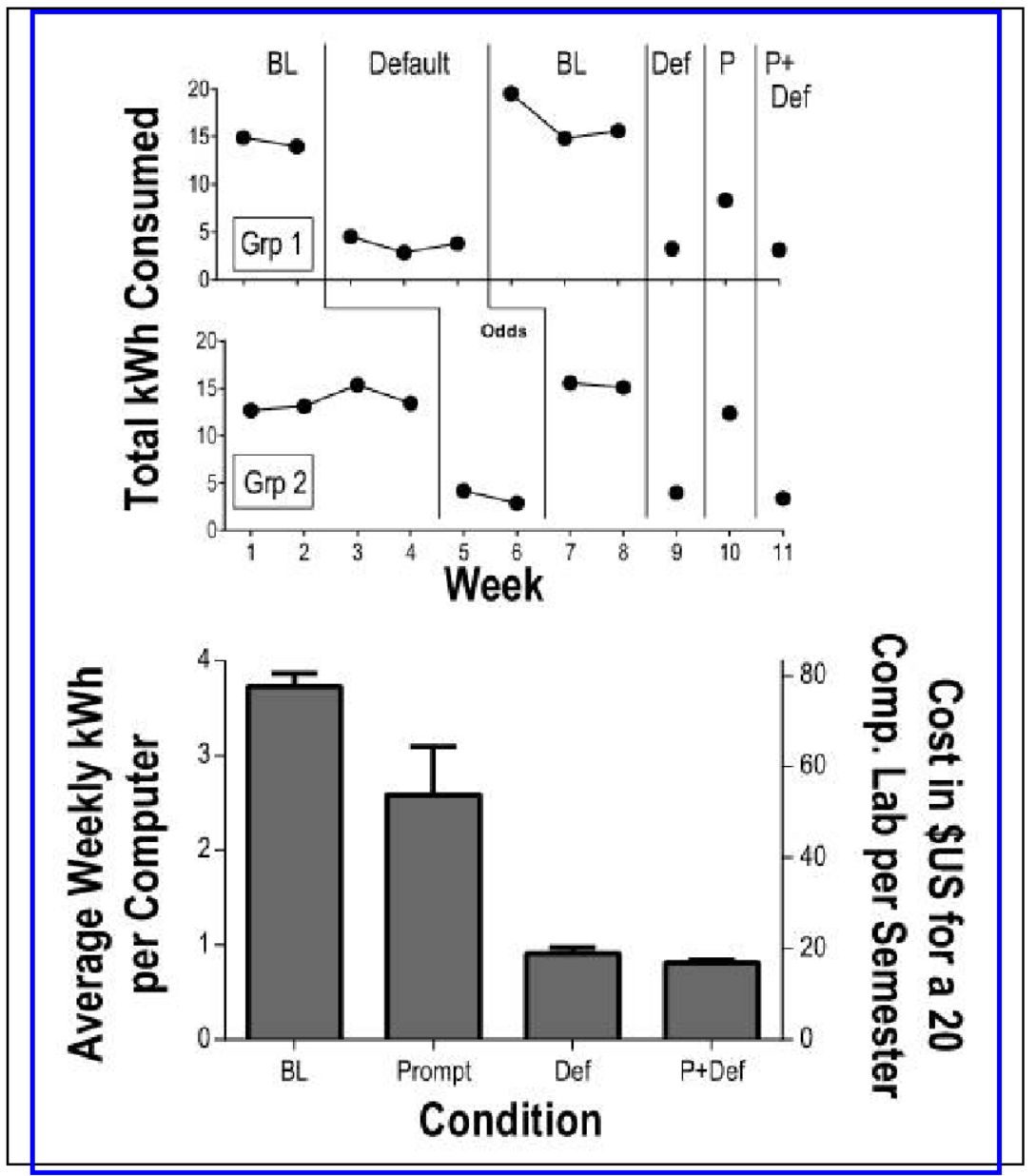

Figure 1. Top panel depicts kWh of energy consumed per week during Baseline (BL), Default (Def), Prompt ( $\mathrm{P}$ ) and Prompt+Default ( $\mathrm{P}+$ Def ) conditions for four computer dyads (two dyads per group). Bottom panel depicts average weekly kWh consumption per dyad for each condition, plotted on the left $y$-axis; error bars represent the standard error of the mean (SEM). The right $y$-axis in the bottom panel represents the cost associated with each condition, extrapolated across an entire academic semester for a 20-computer laboratory,

the testing lab when the power management settings were adjusted such that the computers entered standby mode automatically, without requiring an active response from students. During the default condition, the researchers changed the computers' power management settings such that the monitor would enter standby mode after 10 minutes of inactivity and the computer would enter standby mode after 20 minutes of inactivity. No other changes were made. Students or lab assistants could also manually set the computer to standby mode earlier than the programmed intervals. Despite the freedom to do so, systematic checks revealed that the set- tings were never changed by the participants.

Prompt Condition. In order to evaluate whether a textual prompt would result in students manually placing computers in standby mode when finished with their quizzes, the researchers changed the desktop background of the computers to display in white text on a black background: "Please set the computer to sleep/standby when finished. Thank you." During the baseline and defaults conditions, the desktop background was solid black with no text. During this condition, the researchers returned the power settings to those of the baseline condition. 
Prompt+Default Condition. The purpose of this condition was to determine whether the combination of a textual prompt and the defaulting intervention would produce lower levels of power consumption than either intervention alone.

Procedural Fidelity. The Watts Up? PRO meters were chosen based on a previous paper that validated the device's reliability. ${ }^{11}$ Because data were collected using an automated device with established reliability, the data were not at risk for reliability concerns. Procedural fidelity data were collected on the power management settings on the lab computers. During 50 percent of data extractions, two independent observers recorded the power settings on the computers to ensure they had been set properly and that they had not been changed by users of the computers. These checks revealed that computer settings were set accurately and according to the research protocol (i.e., procedural fidelity was 100 percent).

\section{Results and Discussion}

The top panel of Figure 1 depicts the effects of intervention components during the course of the semester. During baseline, the computers in Group 1 consumed an average of $14.43 \mathrm{kWh} /$ week; Group 2 averaged $13.66 \mathrm{kWh} /$ week. During the default condition, a substantial decrease in the amount of energy consumed was observed in both groups, with Group 1 and Group 2 consuming an average of $3.59 \mathrm{kWh} /$ week and 3.69 $\mathrm{kWh} /$ week, respectively. The levels were replicated in both groups during a reversal to baseline and when a probe of the default condition showed a return to low levels of energy consumption. Implementation of the textual prompt alone resulted in a lower level of energy consumption than the baseline condition, but higher levels than the default condition. During the prompt+default condition, energy consumption did not significantly differ from the default condition, $t(7)=.78, p=.46$, suggesting that the addition of the textual prompt had a minimal effect on energy consumption and may not be necessary in application. Further, these results are consistent with our hypotheses about the importance of response effort in sustainability interventions, specifically regarding the necessity of minimizing effort required of end-users.

The bottom panel of Figure 1 depicts an analysis of the costs associated with rates of energy consumption for each condition. Baseline levels of energy consumption indicated an average of $14.92 \mathrm{kWh} /$ week across the four dyads $(S E M=.55)$. Additionally, the baseline condition consumed more energy than any other condition in the study. Extrapolating this rate of energy consumption across an entire 16-week semester suggests a cost of approximately $\$ 77.59$ to power the 20-computer testing laboratory. In contrast, the least energy consumptive conditions were those featuring the default setting manipulation (default and prompt+default), averaging only 3.55 $\mathrm{kWh} /$ week per computer dyad (SEM = .20 ; extrapolated cost $=\$ 16.28$ for the testing laboratory for an entire semester). This constitutes a savings of 79.02 percent, which translates to a savings of $\$ 61.31$. Note that this is a conservative estimate based on a four-day week. Factoring in continued energy use during Fridays and weekends during baseline yields more dramatic savings.

Perhaps the most important contribution of this study was the resource-efficient means in which energy conservation was obtained. Previous approaches to energy conservation have relied upon intense use of resources (e.g., money, time, effort, or technology). For example, Hayes and Cone ${ }^{13}$ sent energy consumers individualized statements via letter each month; these statements were in addition to a separate utility bill, requiring the use of extra paper and much effort on the part of the change agents and the consumers themselves, who had to open and read the information to subsequently alter usage patterns. Slavin and colleagues ${ }^{14}$ implemented a similar feedback procedure to modify the energy use in master-metered apartments. One notable difference, however, was the integration of a financial incentive to groups of residents with the most savings. Thus, this form of intervention required a substantial investment of both time and monetary resources. As a final example, consider the work by Winett and colleagues ${ }^{15}$ who used group discussions and video models to teach energy conservation skills involving home heating/cooling to participants, in addition to daily individualized written feedback on performance and graphs of savings posted next to thermostats. While effective, this procedure is incredibly resource-intensive and thereby unlikely to sustain over time or be scaled up to community-wide approaches. In each of the foregoing approaches, change agents must (a.) spend a significant amount of time monitoring individual consumers, (b.) provide individualized feedback to consumers, (c.) consume more resources in the provision of paper letters in the delivery of said feedback, and, in some circumstances, (d.) supplement feedback with monetary incentives to motivate behavioral change. For these reasons, it is unlikely that these methods could be implemented successfully at a much larger scale (e.g., on a university campus with many thousands of consumers and energy consuming devices). The authors of the aforementioned studies made attempts to reduce the expense of implementing their methods by reducing the frequency of feedback, ${ }^{13}$ training consumers to construct their own feedback, ${ }^{16}$ and delivering feedback mechanically. ${ }^{17}$ However, by harnessing modern technology, the ongoing need for the involvement of researchers or other change agents can be eliminated. Our study demonstrates efficient use of contemporary technologies to circumvent the need for resource-intensive approaches. The intervention need only be applied by IT professionals who control the default settings applied to computer workstations. This intervention would require no more time than what is already spent in the installation of new machines, resulting in a substantially resource-efficient approach.

We can conclude that strategic energy savings settings can dramatically reduce energy consumption. A notable finding was that the energy saving settings were never altered by the participants, despite potentially more effort in having to wake the computer from sleep during 
energy savings conditions and log back on to the machine.

Two substantial limitations remain which limit these interpretations. First, the prompt condition never followed the baseline condition. Since experimental control in the multiple-baseline time-series design would require this sequence to have occurred, sufficient comparisons between these conditions cannot be made. Second, only the prompt condition influenced participants' energy efficient behaviors (i.e., manually setting the computers to a low-power mode or altering the computer settings) without the aid of energy savings settings. The aggregate data from the prompt condition suggested a moderate improvement over baseline levels, but a substantial further improvement $(36 \%)$ in energy savings under the default condition. Furthermore, extended exposure to the prompt was not evaluated, which may have resulted in decrements in energy savings over time as computer users experienced increased effort or habituated to the presence of the prompt.

Future research might also evaluate the effects of differently constructed prompts to determine whether more graphically complex prompts could result in a more pronounced savings. However, the aim of this study was to demonstrate that while the computer settings alone significantly improved energy consumption, this was achieved without necessarily changing any participants' behaviors. A drawback to this method is that the only way these energy savings can generalize across settings is to specifically set the computers to do so. Notwithstanding these limitations, the current findings represent a necessary first approach in identifying ways to intervene on energy consumption in computer-dense settings, such as universities and office buildings. This study contributes to the growing body of evidence that effort-based interventions are viable means for producing marked change in sustainable behavior.

\section{Author Disclosure Statement}

No conflicting financial interests exist for any author of this report.

\section{References}

1. Tuso MA, and Geller ES. Behavior analysis applied to environmental/ecological problems: A review. J Appl Behav Anal 1976;9:526.

2. Staats $H$, van Leeuwen E, and Wit A. A longitudinal study on informational interventions to save energy in an office building. J Appl Behav Anal 2000;33:101-104.

3. U.S. Department of Energy. Computers and Photocopiers in Commercial Buildings (1999 CBECS data). Energy Information Administration, Washington, DC, 2002. http://www.eia.doe. gov/emeu/cbecs/pc_copier/pccopier99. html (last accessed 11/07/2013).

4. Webber CA, Roberson JA, McWhinney MC, et al. After-hours power status of office equipment in the USA. Energy 2006;31:2823-2838.

5. Christensen KJ, Gunaratne C, Nordman $\mathrm{B}$, et al. The next frontier for communications networks: Power management. Comput Commun 2004;27:1758-1770.

6. Steg L. Promoting household energy conservation. Energy Policy 2008;36:4449-4453.

7. Gifford R. The dragons of inaction: Psychological barriers that limit climate change mitigation and adaptation. Am Psychol 2011;66:290-302.

8. Friman PC, and Poling A. Making life easier with effort: Basic findings and applied research on response effort. J Appl Behav Anal 1995;28:583-590.

9. Thaler RH, and Sunstein, CR. Nudge: Improving decisions about health, wealth, and happiness. Penguin Books, New York, 2009.

10. Van Raaij WF, and Verhallen TMM. A behavioral model of residential energy use. J Econ Psychol 1983;3:39-63.

11. Hirst JM, Miller JR, Kaplan BA, et al. Watts Up? PRO AC Power Meter for automated energy recording: A product review. Behav Anal Pract 2013;6:82-95. 12. Barlow DH, Nock MK, and Hersen M. Single Case Experimental Designs: Strategies for Studying Behavior Change. Pearson Education, Inc., Boston, MA, 2009.

13. Hayes SC, and Cone JD. Reduction of residential consumption of electricity through simple monthly feedback. J Appl Behav Anal 1981;14:81-88.

14. Slavin RE, Wodarski JS, and Blackburn BL. A group contingency for electricity conservation in master-metered apartments. J Appl Behav Anal 1981;14:357-363.

15. Winett RA, Hatcher JW, Fort TR, et al. The effects of videotape modeling and daily feedback on residential electricity conservation, home temperature and humidity, perceived comfort, and clothing worn: Winter and summer. J Appl Behav Anal 1982;15:381-402.

16. Winett RA, Neale MS, and Grier HC. The effects of self-monitoring and feedback on residential electricity consumption: Winter. J Appl Behav Anal 1979;12:173-184.

17. McClelland L, and Cook WS. Energy conservation effects of continuous in-home feedback in all-electric homes. J Environ Syst 1980;9:169-173.

Address correspondence to:

Derek D. Reed, PhD, BCBAD

Department of Applied

Behavioral Science

University of Kansas

4001 Dole Human Development Center

1000 Sunnyside Avenue

Lawrence, KS 66045-7555

E-mail: dreed@ku.edu 